\title{
Bayesian Convolutional Neural Networks for Seismic Facies Classification
}

\author{
Feng, Runhai; Balling, Niels; Grana, Dario; Dramsch, Jesper Soren; Hansen, Thomas Mejer
}

Published in:

IEEE Transactions on Geoscience and Remote Sensing

Link to article, DOI:

10.1109/TGRS.2020.3049012

Publication date:

2021

Document Version

Peer reviewed version

Link back to DTU Orbit

Citation (APA):

Feng, R., Balling, N., Grana, D., Dramsch, J. S., \& Hansen, T. M. (2021). Bayesian Convolutional Neural Networks for Seismic Facies Classification. IEEE Transactions on Geoscience and Remote Sensing, 59(10), 8933 - 8940. https://doi.org/10.1109/TGRS.2020.3049012

\section{General rights}

Copyright and moral rights for the publications made accessible in the public portal are retained by the authors and/or other copyright owners and it is a condition of accessing publications that users recognise and abide by the legal requirements associated with these rights.

- Users may download and print one copy of any publication from the public portal for the purpose of private study or research.

- You may not further distribute the material or use it for any profit-making activity or commercial gain

- You may freely distribute the URL identifying the publication in the public portal 


\title{
Bayesian Convolutional Neural Networks for Seismic Facies Classification
}

\author{
Runhai Feng ${ }^{\circledR}$, Niels Balling, Dario Grana ${ }^{\circledR}$, Jesper Sören Dramsch ${ }^{\circledR}$, and Thomas Mejer Hansen
}

\begin{abstract}
The seismic response of geological reservoirs is a function of the elastic properties of porous rocks, which depends on rock types, petrophysical features, and geological environments. Such rock characteristics are generally classified into geological facies. We propose to use the convolutional neural networks in a Bayesian framework to predict facies based on seismic data and quantify the uncertainty in the classification. A variational approach is adopted to approximate the posterior distribution of neural parameters that is mathematically intractable. The network is trained on labeled examples. The mean and the standard deviation of the distribution of neural parameters are randomly drawn from predefined Gaussian functions for the initialization, and are updated by minimizing the negative evidence lower bound. The facies classification is applied to seismic sections not included in the training data set. We draw multiple random samples from the trained variational posterior distribution to simulate an ensemble predictor and classify the most probable seismic facies. We implement the proposed network in the open-source library of TensorFlow Probability, for its convenience and flexibility. The applications show that the internal regions of the seismic sections are generally classified with higher confidence than their boundaries, as measured by the predictive entropy that is calculated based on a multiclass probability across the possible facies. A plain neural network is also applied for comparison, by assigning fixed values to the neural parameters using a classical backpropagation technique. The comparison shows consistent results; however, the proposed approach is able to assess the uncertainty in the predictions.
\end{abstract}

Index Terms-Bayesian convolutional neural networks, seismic facies classification, uncertainty quantification, variational approach.

\section{INTRODUCTION}

$\mathbf{S}$ EISMIC facies classification aims at predicting subsurface depositional sequences and lateral variations from seismic data [1] to determine favorable reservoir conditions for hydrocarbon and geothermal productions [2], [3]. Conventional workflows including horizon interpretation, petrophysical analysis, and geological modeling are usually applied.

Manuscript received September 18, 2020; revised December 7, 2020; accepted December 29, 2020. This work was supported by the Innovation Fund Denmark under Grant 6154-00011B. (Corresponding author: Runhai Feng.)

Runhai Feng, Niels Balling, and Thomas Mejer Hansen are with the Department of Geoscience, Aarhus University, 8000 Aarhus, Denmark (e-mail: r.feng@geo.au.dk).

Dario Grana is with the Department of Geology and Geophysics, University of Wyoming, Laramie, WY 82071 USA.

Jesper Sören Dramsch is with the Danish Hydrocarbon Research and Technology Centre, Technical University of Denmark, 2800 Kongens Lyngby, Denmark.

Color versions of one or more figures in this article are available at https://doi.org/10.1109/TGRS.2020.3049012.

Digital Object Identifier 10.1109/TGRS.2020.3049012
Stratigraphic boundaries are picked along seismic interfaces, and facies sequences are classified based on the characteristics of seismic response (e.g., amplitude variations). In this context, the term facies may refer to any possible geological body of rocks based on different lithological, depositional, or geophysical properties.

Because of the convolutional nature of the seismic signal, the classification cannot be performed independently at each point location, and the seismic data depend on the elastic contrast at the interface of different facies transitions. Manual classification of seismic facies is typically time-consuming and heavily relies on the expertise of skilled interpreters [4]. Inversion methods for these categorical/discrete variables can then be adopted to solve this classification problem based on seismic data. Several solutions have been proposed, including statistical learning methods, such as the support vector machine [5], the $K$-nearest neighbor [6], the self-organizing maps [7], the random forest [8], the independent component analysis [9], and the generative topographic mapping [10].

Deep learning methods, such as convolutional neural networks (CNN), can provide accurate and efficient tools to predict higher-level features from input signals automatically. Many approaches have been proposed for seismic facies classification using deep learning [11]-[13]. For example, Grana et al. [14] compared deep learning algorithms to the Monte Carlo methods for facies classification from seismic data. Zhang et al. [15] classified seismic facies in stratigraphic sequences based on deep learning. To overcome the requirement in a large volume of labeled examples, semisupervised generative adversarial networks [16] and unsupervised schemes [17] have been proposed. To analyze the uncertainty in the predictions, different methods have been developed. Das et al. [18] applied an approximated Bayesian computation method to estimate the posterior distribution of predictions obtained by the CNN. Liu and Grana [19] adopted an ensemble-based method for a geostatistical inversion to evaluate the associated uncertainty.

However, the aforementioned methods do not account for the uncertainty in the deep learning parameters, and only a single value is provided by applying simple activation functions, given each input feature [11]-[17]. This is because, in a typical implementation of a neural network, the weight and the bias of a neuron are assumed to be deterministic. Training such a network again provides a new prediction due to different initialized values in neural parameters. The uncertainty related to the neural network itself is not explicitly formulated and accounted for [20]. 


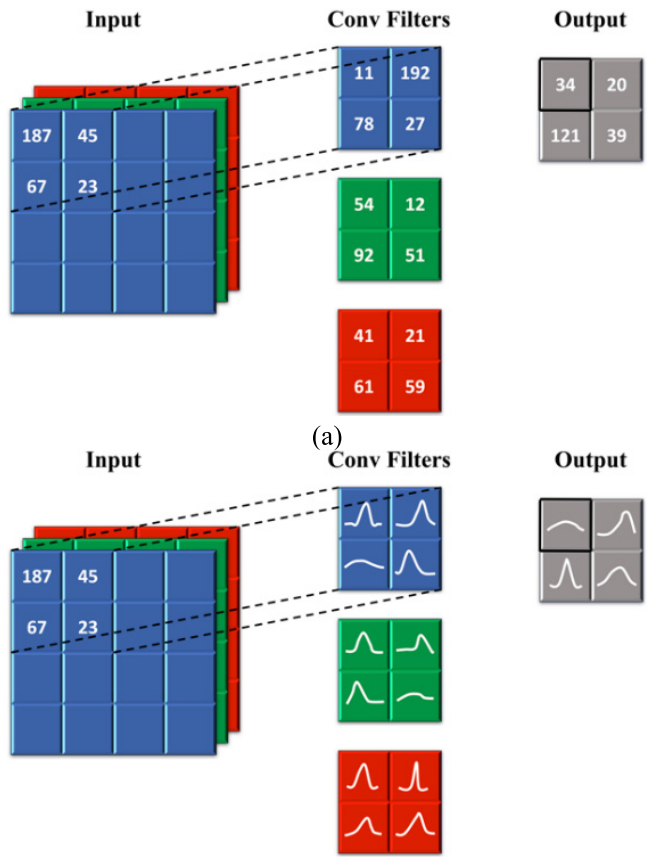

(b)

Fig. 1. (a) Plain neural networks (CNN-based) with fixed values of the neural parameters after training on labeled examples. (b) BCNN with independent probability distributions of the neural parameters.

The application to seismic data poses several challenges due to the presence of random noises, imaging artifacts, and limited training examples, which makes the facies classification nonunique. Therefore, geological interpretation, risk analysis, and decision-making processes based on a seismic facies model require accurate uncertainty quantification studies. Ghahramani [20] described a probabilistic framework for investigating the uncertainty of models and predictions in the learning process. A common strategy for uncertainty quantification in deep learning methods is the Monte Carlo dropout sampling [21], [22].

In this article, we propose a Bayesian approach for the CNN method to classify facies from seismic data. The method is based on a variational scheme for the approximation of the posterior distribution of neural parameters [23]. The Bayesian inference problem is formulated as an optimization process through the variational inference (VI) method, and the network is trained using a stochastic gradient approach. Bayesian networks allow for rigorous estimation of the uncertainty of the predictions by updating the parameters of a variational function. After training, multiple random samples are drawn from the trained posterior distribution to simulate an ensemble predictor, and the uncertainty in the classification is evaluated using the prediction entropy. This approach differs from traditional plain neural network methods that only provide deterministic estimates and often lead to overconfident predictions in regions with little or no training examples [24].

\section{THEORY}

CNN models are designed for image segmentation at the pixel level [25], [26]. In a typical training process, neural parameters in the CNN are updated and fixed by learning on labeled examples [see Fig. 1(a)], which means that only a

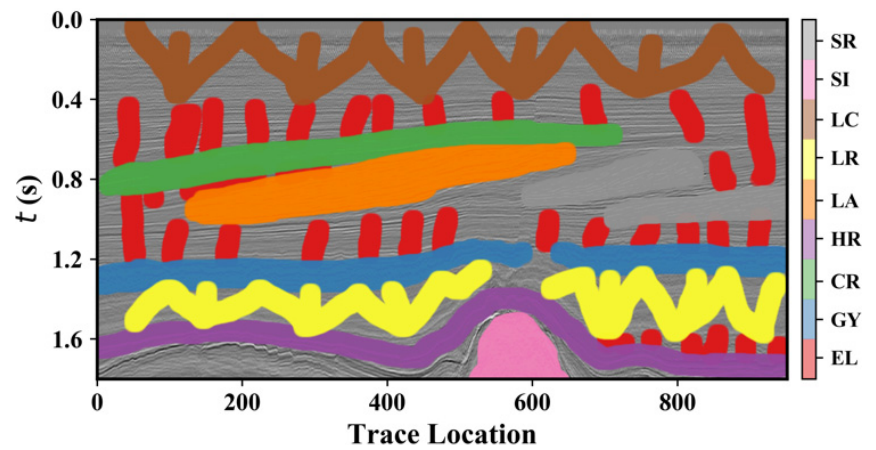

Fig. 2. Seismic facies classified on a training image [13]. We refer to the main text for different seismic facies definitions.

single set of values is obtained given each input feature. This approach, often referred to as plain neural networks, fails to quantify the uncertainty in the predictions [see Fig. 1(a)].

Instead, we introduce a probability distribution of the neural parameters using the Bayes' theorem [22] and propose a Bayesian CNN (BCNN). This approach can be viewed as a probabilistic model capable of quantifying the posterior uncertainty [see Fig. 1(b)]. Let $p(\omega \mid D)$ indicate the posterior distribution of neural parameters $\omega$, given the training data $D$; then, the probability of the model prediction $y^{*}$ for a test sample $x^{*}$ is obtained by integrating over all possible values in $\omega$

$$
p\left(y^{*} \mid x^{*}, D\right)=\int p\left(y^{*} \mid x^{*}, \omega\right) p(\omega \mid D) d \omega
$$

in which $p\left(y^{*} \mid x^{*}, \omega\right)$ is the probability of $y^{*}$ given $x^{*}$ and $\omega$.

The probability distribution $p(\omega \mid D)$ is mathematically intractable for any practical size of neural networks [24]. Sampling methods, such as the Markov chain Monte Carlo (MCMC), can be adapted to approximate the distribution $p(\omega \mid D)$, but they are computationally demanding due to the sampling in high-dimensional space [27]. Alternatively, $p(\omega \mid D)$ can be approximated using a variational distribution $q_{\theta}(\omega)$ that can be easily assessed and allows for efficient learning of the network parameters [27]. This approach is referred to as VI, and it is more efficient than MCMC [28]. In the VI, the proposed distribution should be as close as possible to the posterior distribution of the neural parameters obtained from the original model [24]. We use the Kullback-Leibler (KL) divergence to measure the similarity between the two distributions and achieve the optimal variational parameter $\theta^{\text {opt }}$ through a minimization process

$$
\begin{aligned}
\theta^{\text {opt }}= & \underset{\theta}{\arg \min } \operatorname{KL}\left[q_{\theta}(\omega) \| p(\omega \mid D)\right] \\
= & \underset{\theta}{\arg \min } \operatorname{KL}\left[q_{\theta}(\omega) \| p(\omega)\right] \\
& -\mathrm{E}_{q_{\theta}(\omega)}[\log p(D \mid \omega)]+\log p(D)
\end{aligned}
$$

where $p(\omega)$ is the prior distribution of $\omega$, and $\mathrm{E}$ denotes the expectation. The term $\log p(D)$ is also intractable but, because it is constant, it can be omitted in the optimization procedure [24]. Minimizing $\operatorname{KL}\left[q_{\theta}(\omega) \| p(\omega \mid D)\right]$ is equivalent to maximizing $\mathrm{E}_{q_{\theta}(\omega)}[\log p(D \mid \omega)]-\mathrm{KL}\left[q_{\theta}(\omega) \| p(\omega)\right]$, which can be referred to as the evidence lower bound (ELBO). 


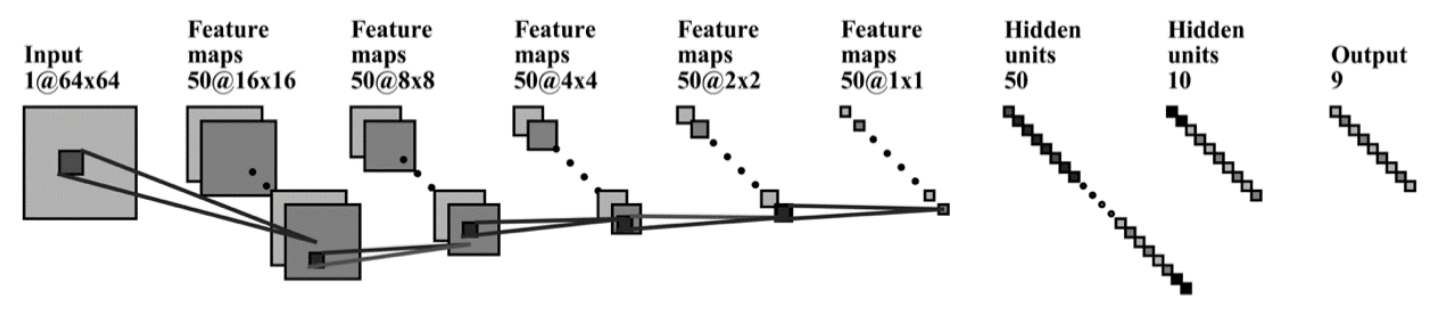

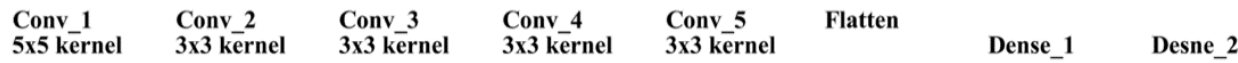

Fig. 3. Network architecture, in which there are five convolutional and two dense layers.

The loss function in the BCNN is then defined as in [27]

$$
\mathcal{F}(D, \theta)=\mathrm{KL}\left[q_{\theta}(\omega) \| p(\omega)\right]-\mathrm{E}_{q_{\theta}(\omega)}[\log p(D \mid \omega)]
$$

where the first term $\operatorname{KL}\left[q_{\theta}(\omega) \| p(\omega)\right.$ is the divergence between variational and prior distributions, and the second term $\mathrm{E}_{q_{\theta}(\omega)}[\log p(D \mid \omega)]$ is the likelihood that describes how the variational distributions of the neural parameters explain the observed data [28]. Thus, the objective function in (3) depends on the likelihood and the prior terms. The variational function is not a function of the training data $D$. The loss in (3) and its corresponding KL minimization relate the variational distribution to the training data [28].

As in the mean-field VI scheme, a Gaussian distribution with mean $\mu$ and standard deviation $\sigma$ is adopted as the variational distribution

$$
q_{\theta}(\omega)=\mathcal{N}\left(\omega \mid \mu, \sigma^{2}\right)
$$

The parameters $\mu$ and $\sigma$ are learned by optimizing the loss function in (3) using the stochastic gradient approach [28], [29]. Hence, neural parameters $\omega$ are then represented by independent Gaussian functions with different $\mu$ and $\sigma$. Compared to a plain CNN [see Fig. 1(a)], the number of trainable parameters in the BCNN [see Fig. 1(b)] is larger due to the parameterization of the distribution.

In practical applications, the training data $D$ are often randomly split into a partition of $M$ subsets of equal size for a minibatch optimization [23], and (3) is modified to account for the loss gradient that is averaged over the minibatches [30]

$$
\mathcal{F}_{i}\left(D_{i}, \theta\right)=\frac{1}{M} \mathrm{KL}\left[q_{\theta}(\omega) \| p(\omega)\right]-\mathrm{E}_{q_{\theta}(\omega)}\left[\log p\left(D_{i} \mid \omega\right)\right]
$$

for $i=1, \ldots, M$, where $D_{i}$ is the data in the minibatch $i$ and $\sum_{i} \mathcal{F}_{i}\left(D_{i}, \theta\right)=\mathcal{F}(D, \theta)$. We implement the proposed BCNN on the open-source platform of TensorFlow Probability [see Fig. 1(b)], but it could also be included in other machine learning packages. The proposed implementation uses the Flipout gradient estimator [31] to minimize the loss function in (5), in which a stochastic forward pass is applied by sampling the posterior distribution of neural parameters. The stochastic gradient descent method is used to update the neural parameters, and its performance depends on the variance of the gradient [27]. Due to a large number of neural parameters, all training examples in each minibatch share the same perturbation scheme. Thus, the variance of the estimator cannot be reduced effectively [31]. The Flipout can

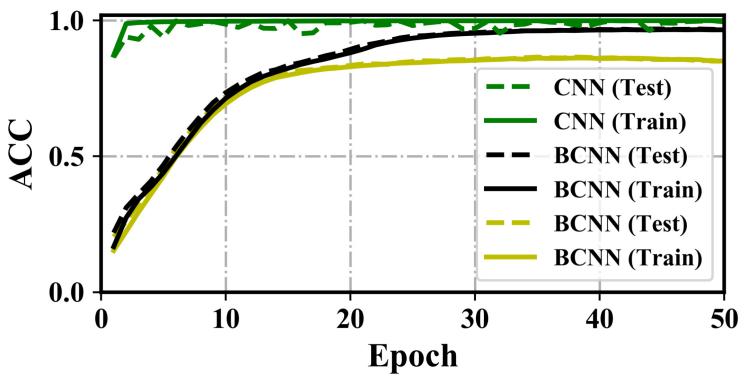

Fig. 4. Accuracy (ACC) of training (solid curve) and testing (dashed curve) data sets. CNN results are in green, and BCNN results of the reference case $\left[p(\omega)=\mathcal{N}\left(0,10^{2}\right)\right]$ are in black. Yellow curves show BCNN results with a different prior $\left[p(\omega)=\mathcal{N}\left(0,1^{2}\right)\right]$.

efficiently de-correlate the gradients within the minibatches (5) by applying pseudo-independent parameter perturbations for each input [29], [31]. A linear reduction in the variance is then expected for complex network models, such as convolutional or recurrent networks.

To make predictions using the BCNN given input features, Monte Carlo samples are drawn from the trained variational posterior distribution $q_{\theta}(\omega)$, leading to an ensemble predictor. For a general inverse problem, the expectation E can be approximated by averaging the Monte Carlo estimates

$$
\mathrm{E}_{q_{\theta}(\omega)}\left[p\left(y^{*} \mid x^{*}, \omega\right)\right] \approx \frac{1}{T} \sum_{t=1}^{T} p\left(y^{*} \mid x^{*}, \hat{\omega}_{t}\right)
$$

where $T$ is the number of random samples (i.e., stochastic forward pass) and $\hat{\omega}_{t}$ is the sampled neural parameter. However, for a classification problem of categorical variables, such as seismic facies classification, we introduce an indicator function $I(\cdot)$ to predict the categorical labels

$$
L=\underset{c}{\arg \max } \sum_{t=1}^{T} I\left(\underset{c}{\arg \max } p\left(y^{*}=c \mid x^{*}, \hat{\omega}_{t}\right)\right)
$$

in which $p\left(y^{*}=c \mid x^{*}, \hat{\omega}_{t}\right)$ is the softmax categorical probability of label $c$ given input $x^{*}$ and sampled neural parameter $\hat{\omega}_{t}$. Therefore, $L$ is the most-likely prediction based on the random samples drawn from the trained networks.

In addition to the most-likely prediction, the $\mathrm{BCNN}$ allows quantifying the posterior uncertainty, which can be evaluated 


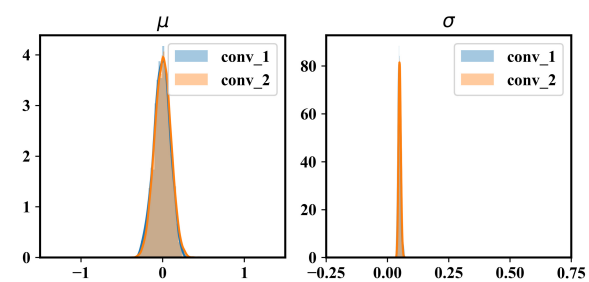

(a)

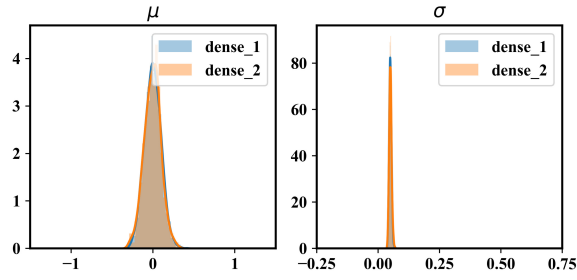

(d)

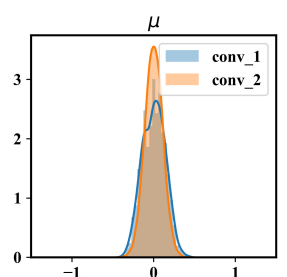

(b)

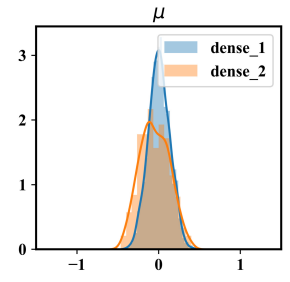

(e)
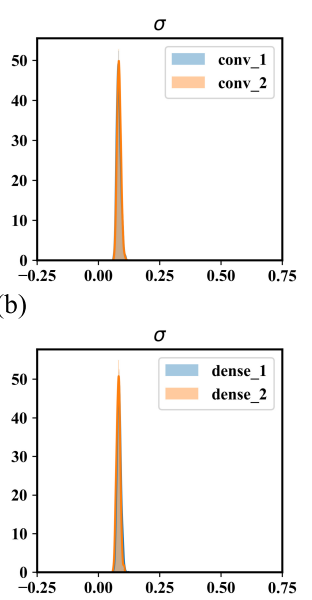

(e)
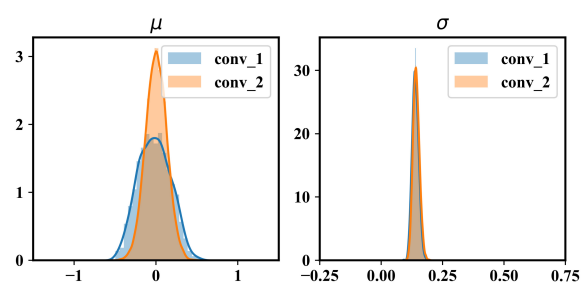

(c)
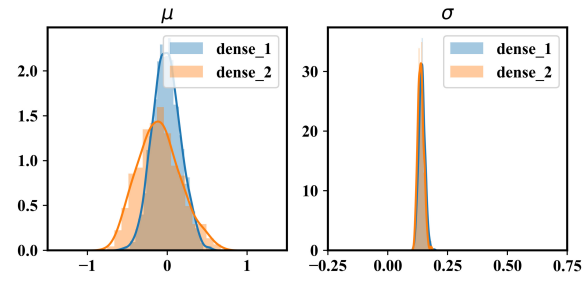

(f)

Fig. 5. Univariate distributions of posterior neural parameters $\mu$ and $\sigma$ of (a)-(c) convolutional layers and (d)-(f) dense layers for (a) and (d) epoch 1, (b) and (e) epoch 25, and (c) and (f) epoch 50.

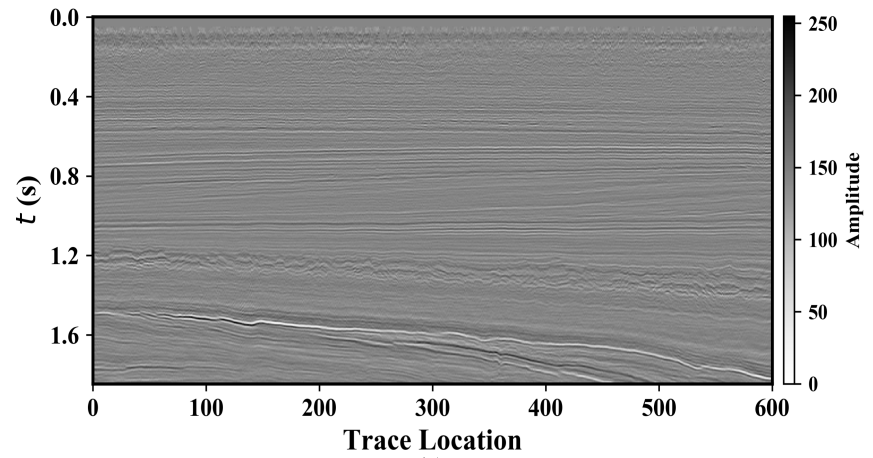

(a)

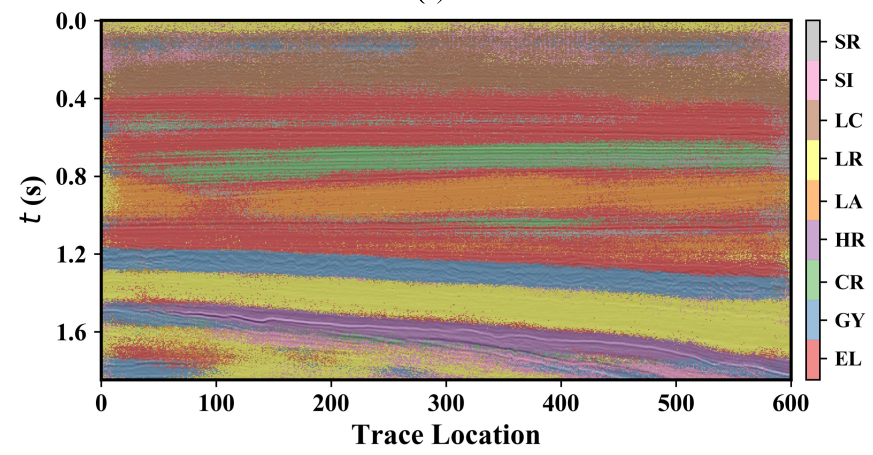

(c)

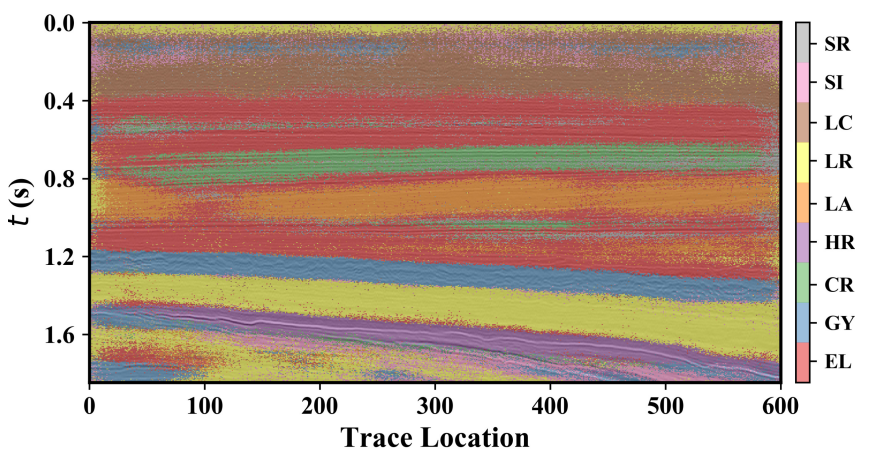

(b)

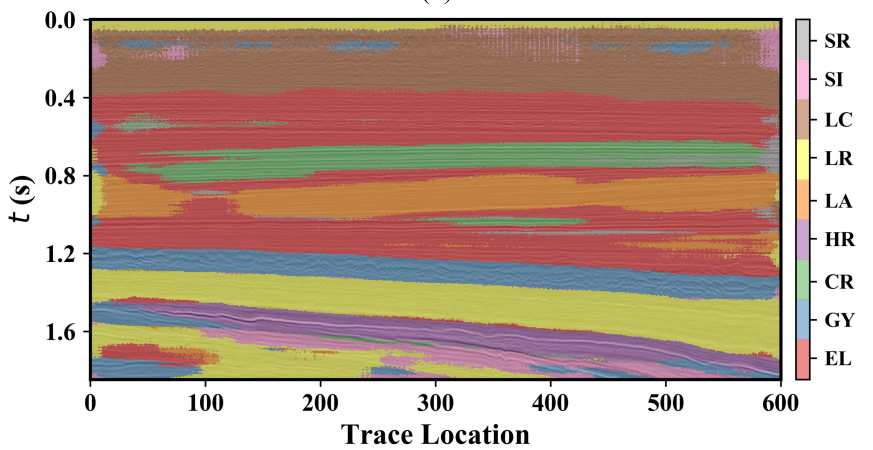

(d)

Fig. 6. First example. (a) Input seismic section. (b) and (c) Two randomly selected facies realizations. (d) Classification based on 100 ensemble predictions.

using the predictive entropy [26]

$$
\begin{aligned}
& \tilde{H}\left[y^{*} \mid x^{*}, D\right] \\
& =-\sum_{c=1}^{C}\left(\frac{1}{T} \sum_{t=1}^{T} p\left(y^{*}=c \mid x^{*}, \hat{\omega}_{t}\right)\right) \\
& \quad \times \log \left(\frac{1}{T} \sum_{t=1}^{T} p\left(y^{*}=c \mid x^{*}, \hat{\omega}_{t}\right)\right)
\end{aligned}
$$

where the maximum value is obtained when all predicted labels have equal probabilities (i.e., uniformly uncertain); the minimum value is obtained when one label has a probability equal to 1 and all others have zero probabilities (i.e., highly certain).

To summarize, in this proposed application, the input of the classification is seismic data, whereas the output is a discrete (categorical) variable representing the facies. Facies are defined by geophysicists/geologists based on the geophysical response. The variational approach is used to quantify the classification uncertainty.

\section{ApPLICATION}

The case study is from the Netherlands offshore F3 block seismic data, freely provided by TNO (Netherlands 
Organization for Applied Scientific Research). Nine seismic facies have been identified by seismic interpreters [13], including steep dipping reflectors (SR), salt intrusions (SI), low-coherency regions (LC), low-amplitude regions (LR), low-amplitude dipping reflectors (LA), high-amplitude regions (HR), continuous high-amplitude regions (CR), grizzly amplitude patterns (GY), and everything else (EL) [see Fig. 2].

We adopt a convolutional network architecture introduced by Waldeland and Solberg [32], and we modify it into 2-D for the seismic facies classification. In the 2-D CNN, the layer input is the seismic amplitude data with a patch size of $64 \times 64$ pixels [see Fig. 3]. Nine units are used in the output layer with a softmax function, which corresponds to the seismic facies. Each convolutional and dense-connected layer is followed by the batch normalization and the ReLU activation [25].

In the BCNN [see Fig. 1(b)], we replace all the convolutional and dense layers of the $\mathrm{CNN}$ with their corresponding Flipout layers. All the neural weights in both the convolutional filters and the dense layers are replaced with independent Gaussian distributions, parameterized by mean $\mu$ and standard deviation $\sigma$. The prior $p(\omega)$ is also Gaussian. In the plain CNN [see Fig. 1(a)] used for comparison, a dropout layer is added after the second (Conv_2), third (Conv_3), and fourth (Conv_4) convolutional layers with ratios of 0.5, 0.4, and 0.2, respectively [see Fig. 3]. However, the dropout layer is not required in the $\mathrm{BCNN}$, as the posterior distributions of the neural parameters constitute a regularization term [27].

The categorical cross-entropy is used as the loss function to measure the network model error in the CNN [see Fig. 3]. The number of trainable parameters is 189537 in the BCNN and 95197 in the CNN. The neural biases in both the BCNN and the $\mathrm{CNN}$ are defined as deterministic values.

We train the BCNN and the CNN using the labeled examples in Fig. 2, which are randomly separated into training (75\%, 119109 samples) and testing (25\%, 39703 samples) subsets. For the initialization of the BCNN, the neural weights are parameterized as $\omega=(\mu, \sigma)$. The mean parameters $\mu$ are randomly drawn from $\mathcal{N}\left(0,0.1^{2}\right)$, whereas the standard deviations $\sigma$ are obtained by randomly drawing parameters $\rho$ from $\mathcal{N}\left(-3,0.1^{2}\right)$ and transforming them using a softplus function

$$
\sigma=\log \left(1+e^{\rho}\right)
$$

to ensure nonnegative values. For the initialization of the $\mathrm{CNN}$, the weights are randomly initialized using the Glorot initialization with a uniform distribution [25]. All the biases in the BCNN and the CNN are set to zeros in the initialization [25]. The networks are trained from scratch, and we do not apply the transfer learning with pretrained models [13].

Fig. 4 shows the metrics of accuracy (ACC) of the BCNN and the CNN on the training and testing data sets. We use the T4 GPU (graphics processing unit) in Colaboratory notebooks, executed on Google's cloud servers [33], to train the designed networks. The training time is about $2 \mathrm{~h}$ for the BCNN and about $1 \mathrm{~h}$ for the CNN. The BCNN can achieve accuracy close to $100 \%$ [black curve in Fig. 4] with 50 epochs, whereas the CNN converges faster due to the smaller number of

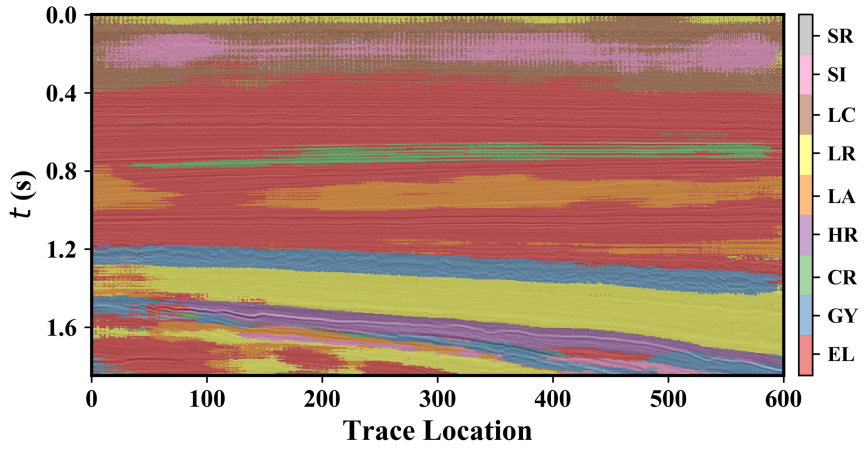

Fig. 7. First example: classified seismic facies by the plain CNN based on the seismic section in Fig. 6(a) as input.

parameters. An early stopping criterion can be used to prevent overfitting the neural models.

We adopt two different priors $p(\omega)$ in the BCNN: the reference case uses a prior with a large initial standard deviation $p(\omega)=\mathcal{N}\left(0,10^{2}\right)$, and we compare the results to those obtained with a prior with a narrower standard deviation $p(\omega)=\mathcal{N}\left(0,1^{2}\right)$. To achieve a comparable accuracy as the $\mathrm{CNN}$, it is preferable to set the prior with a large standard deviation. Indeed, the prior affects the KL divergence of the proposed $q_{\theta}(\omega)$ and prior $p(\omega)$ distributions (5) and further impacts network performance [28]. In the following, we only apply the BCNN with $p(\omega)=\mathcal{N}\left(0,10^{2}\right)$.

Fig. 5 shows the univariate distributions of posterior neural parameters, $\mu$ and $\sigma$, in which a kernel density function is estimated to smooth the histogram. Parameters of the first two convolutional and dense layers are displayed at different training epochs. In the initial training steps, posterior parameters are the values initialized independently according to predefined Gaussian distributions [see Fig. 5(a) and (d)]. With an increase in training epochs, the posterior parameters are updated using the KL divergence optimization. The distributions for epoch 25 and epoch 50 are shown in Fig. 5(b), (c), (e), and (f).

Random samples are then drawn from the trained posterior distributions of neural parameters for the forward pass to generate an ensemble of multiple predictions, based on an input seismic section that is unlabeled (i.e., the seismic data to be classified) shown in Fig. 6(a). Fig. 6(b) and (c) shows two randomly selected realizations by the BCNN. Fig. 6(d) shows the most-likely classified image based on 100 realizations computed in (7). The ensemble classification [see Fig. 6(d)] represents a smooth model, whereas the individual realizations [see Fig. 6(b) and (c)] show higher heterogeneities. Fig. 7 shows the classification obtained by the plain CNN, which is consistent with the BCNN prediction [see Fig. 6(d)]. In the $\mathrm{CNN}$ result [see Fig. 7], more data points are classified as SI facies in the upper part of the section, and the thickness of the CR layer in the middle part is smaller compared to the one by the BCNN [see Fig. 6(d)].

The prediction uncertainty of the BCNN for a different number of realizations is shown in Fig. 8. The internal regions of facies units are classified with a higher confidence (i.e., with smaller entropy values), whereas the facies boundaries are generally uncertain with a larger entropy. In the upper part 


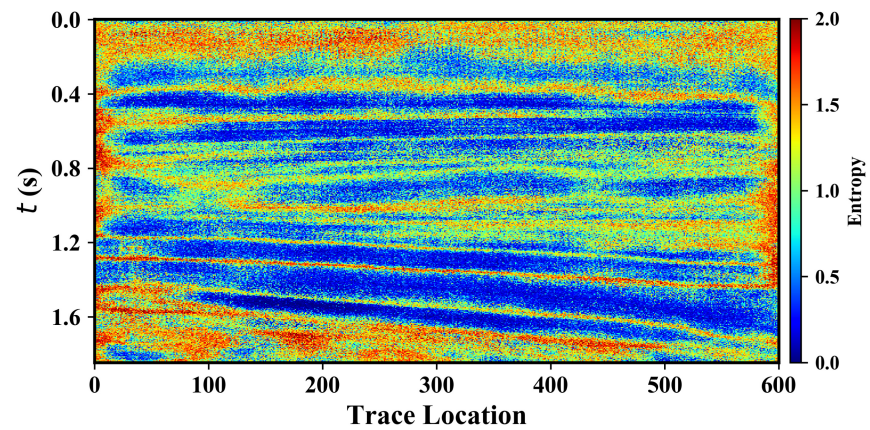

(a)

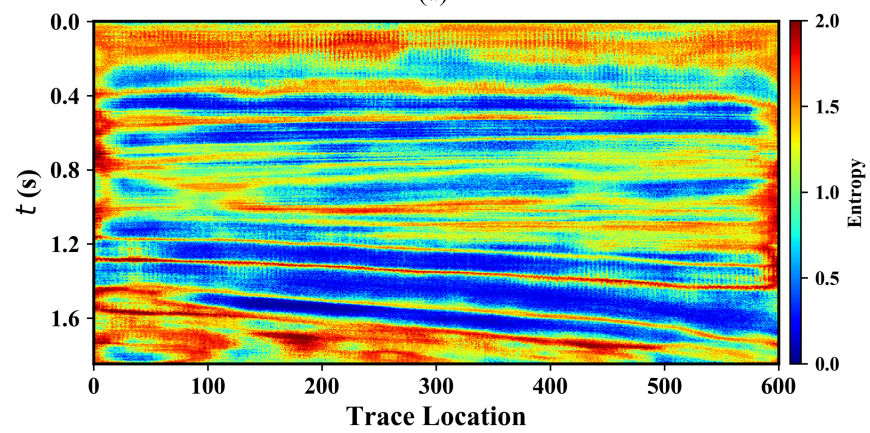

(c)

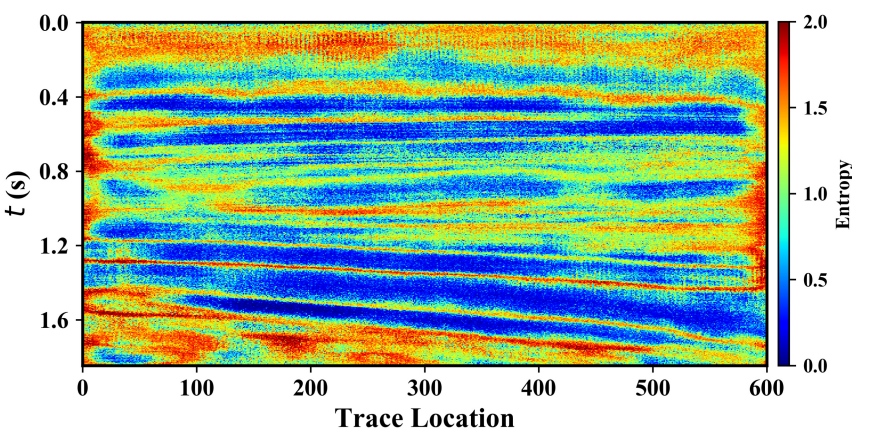

(b)

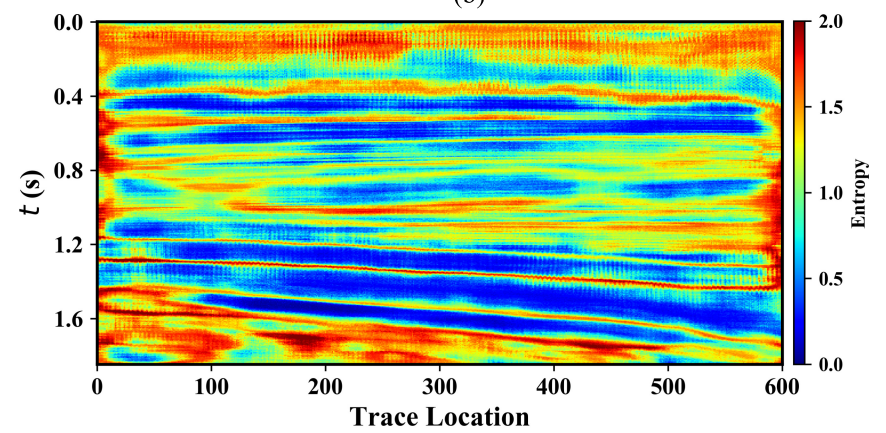

(d)

Fig. 8. First example: prediction entropy of (a) 2, (b) 5, (c) 20, and (d) 100 realizations in the ensemble.

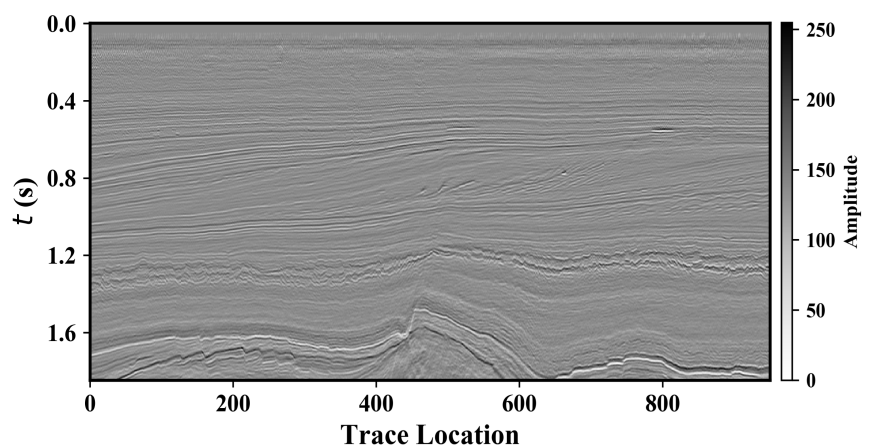

(a)

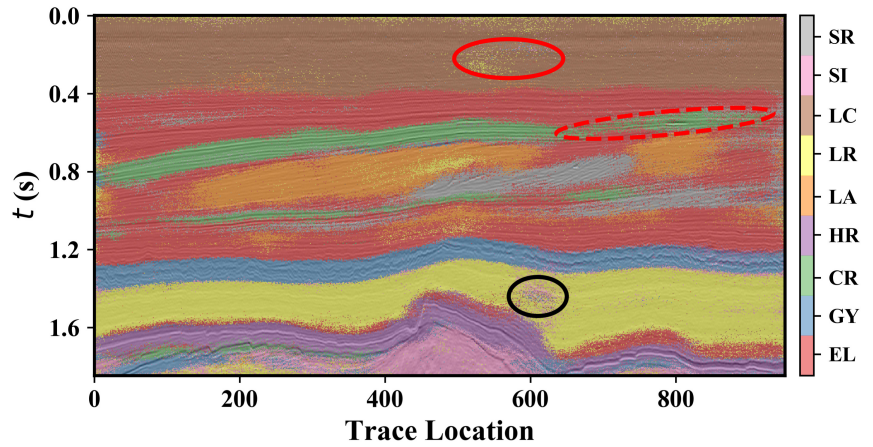

(c)

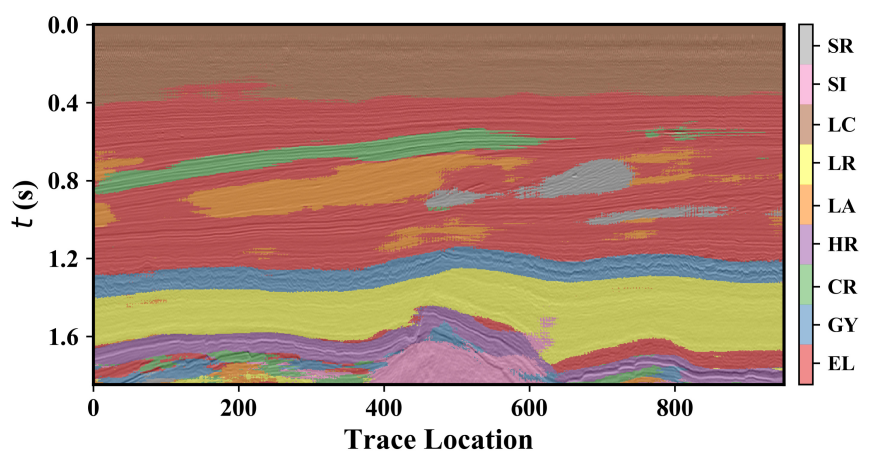

(b)

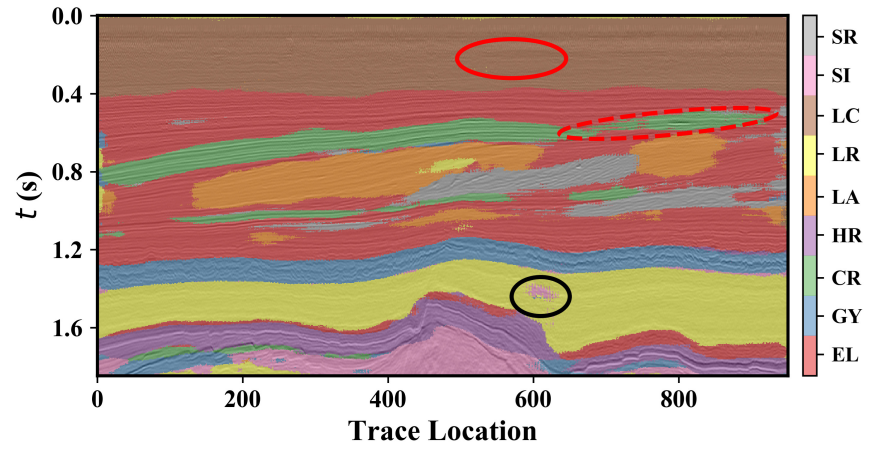

(d)

Fig. 9. Second example. (a) Input seismic section. (b) Facies classification by the CNN. (c) One random facies realization by the BCNN. (d) Classification based on ensemble predictions by the BCNN after 100 realizations.

(at a relative travel time of $0-0.2 \mathrm{~s}$ ), the results show a relatively high entropy value, and the seismic facies classification is uncertain. Indeed, SI, LR, and GY are classified inside the unit of LC facies [see Fig. 6(d)].
We validate our approach to another section of seismic data [see Fig. 9(a)]. Fig. 9(b) shows the classification result by the CNN. The BCNN results are shown in Fig. 9(c) and (d), which includes one random realization and the classification based on 


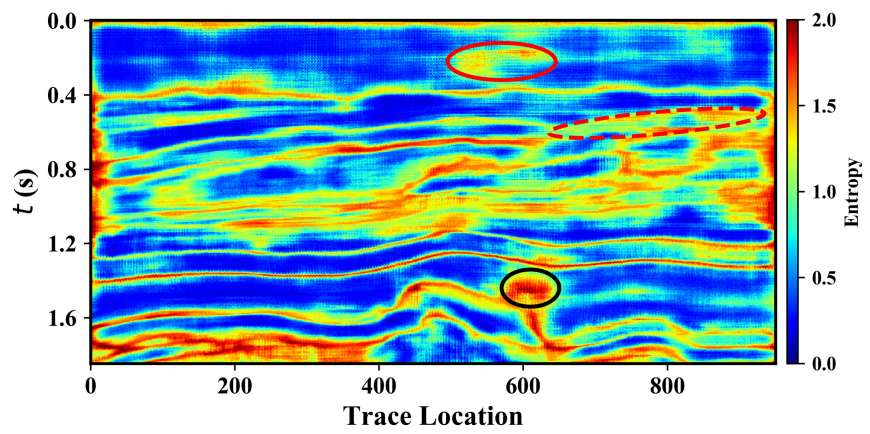

Fig. 10. Second example: prediction entropy of 100 realizations by the BCNN.

ensemble predictions, respectively. Fig. 10 shows the posterior uncertainty based on 100 realizations by the BCNN.

Similar to the results in Fig. 8, high uncertainty is observed at the facies transitions. The uncertainty at $1.5 \mathrm{~s}$ and trace location 600 [marked as a solid black ellipse in Fig. 10] is relatively high and corresponds to the classification of SI facies within the LR unit [solid black ellipse in Fig. 9(c) and (d)]. The CR facies is classified as a continuous layer across the whole section; however, the classification has a higher entropy in some regions [dashed red ellipse in Fig. 10], which suggests possible discontinuities in the layer [dashed red ellipse in Fig. 9(c) and (d)]. A similar result can be observed in the top-middle part [solid red ellipse in Fig. 10], where LR facies is predicted in the individual realization [solid red ellipse in Fig. 9(c)], but LC facies is classified after the ensemble predictions [solid red ellipse in Fig. 9(d)].

\section{DISCUSSION}

To predict the most-likely model and quantify the uncertainty in seismic facies classification, we use a variational scheme to approximate the posterior distribution of neural parameters that are often mathematically not tractable. The proposed approach belongs to the category of VI methods [28], which can be combined with deep learning techniques to analyze the uncertainty in the network predictions. Gaussian functions are used for the approximation of the posterior distributions to derive a full Bayesian network. Mixtures of Gaussian densities can also be adopted for the variational distribution of the neural parameters [23]. Other approaches based on dropout techniques for uncertainty representation in deep learning could be applied as in [21] and [22].

In our application, we replaced all the common convolutional and dense layers with their corresponding variational formulation implemented with the Flipout estimator [31]. Alternative implementations, not investigated in this proposed research, include networks where the shallow layers are represented with deterministic weights, and deep layers are modeled with probabilistic values [24], which leads to shorter training time and fewer memory requirements. Unsupervised approaches in the facies classification [34] could also be explored in future research directions, where larger data sets are considered.

The proposed BCNN has been validated on a seismic facies classification problem with poststack seismic data as inputs. The proposed application assumes that prestack seismic data have been preprocessed and stacked to increase the coherency of the signal. This processing generally includes deconvolution, sorting, velocity analysis, normal-moveout correction, multiple attenuation, dip-moveout correction, and stacking. The seismic classification method can be extended to partial-stacked seismic data commonly used in amplitude versus offset (AVO) studies. The proposed network is to be applied to continuous reservoir properties, such as porosity, mineral, and fluid volumes, using regression processes [35], [36]. Instead of using the softmax for the classification, a sigmoid function must be adopted in the output layer with only one single neuron for the activation.

\section{CONCLUSION}

We presented a deep learning method for the classification of seismic facies where the values of the neural parameters are estimated in a Bayesian setting and the prediction uncertainty is quantified. The posterior distribution of neural parameters is approximated by a variational function, and the optimal parameters are determined by minimizing the loss of negative ELBO to update the variational parameters. As measured by the predictive entropy, the uncertainty of the classification results can be numerically evaluated. The proposed approach is applied to real seismic data from the F3 block (offshore Netherlands) using 2-D neural networks. Generally, the boundaries of seismic facies have high uncertainty as a result of gradual transitions or low resolution of the measured data. High uncertainty within the facies units can be caused by processing errors, data noises, or local heterogeneities. Compared to the plain $\mathrm{CNN}$ that can only provide a deterministic prediction, the proposed BCNN is able to quantify the uncertainty in the predictions, which is important for decision making. The main limitation is that the training time of the BCNN is longer compared to plain $\mathrm{CNN}$ models due to the larger number of trainable model parameters. Then, the tensor processing unit (TPU), application-specific integrated circuits developed by Google, is going to be used to accelerate network learning.

\section{REFERENCES}

[1] F. Li, H. Zhou, Z. Wang, and X. Wu, "ADDCNN: An attention-based deep dilated convolutional neural network for seismic facies analysis with interpretable spatial-spectral maps," IEEE Trans. Geosci. Remote Sens., early access, Jun. 12, 2020, doi: 10.1109/TGRS.2020.2999365.

[2] M. Pang, J. Ba, L.-Y. Fu, J. M. Carcione, U. I. Markus, and L. Zhang, "Estimation of microfracture porosity in deep carbonate reservoirs based on 3D rock-physics templates," Interpretation, vol. 8, no. 4, pp. SP43-SP52, Jul. 2020.

[3] W. Tan, J. Ba, T. Müller, G. Fang, and H. Zhao, "Rock physics model of tight oil siltstone for seismic prediction of brittleness," Geophys. Prospecting, vol. 68, no. 5, pp. 1554-1574, Jun. 2020.

[4] C. E. Bond, A. D. Gibbs, Z. K. Shipton, and S. Jones, "What do you think this is? 'Conceptual uncertainty' in geoscience interpretation," GSA Today, vol. 17, no. 11, pp. 4-10, Nov. 2007.

[5] Y. Nishitsuji and R. Exley, "Elastic impedance based facies classification using support vector machine and deep learning," Geophys. Prospecting, vol. 67, no. 4, pp. 1040-1054, May 2019.

[6] T. Wrona, I. Pan, R. L. Gawthorpe, and H. Fossen, "Seismic facies analysis using machine learning," Geophysics, vol. 83, no. 5, pp. O83-O95, Sep. 2018.

[7] T. Zhao, F. Li, and K. J. Marfurt, "Constraining self-organizing map facies analysis with stratigraphy: An approach to increase the credibility in automatic seismic facies classification," Interpretation, vol. 5, no. 2, pp. T163-T171, May 2017. 
[8] Y. Ao, H. Li, L. Zhu, S. Ali, and Z. Yang, "Identifying channel sandbody from multiple seismic attributes with an improved random forest algorithm," J. Petroleum Sci. Eng., vol. 173, pp. 781-792, Feb. 2019.

[9] D. Lubo-Robles and K. J. Marfurt, "Independent component analysis for reservoir geomorphology and unsupervised seismic facies classification in the taranaki basin, new zealand," Interpretation, vol. 7, no. 3, pp. SE19-SE42, Aug. 2019.

[10] A. Roy, A. S. Romero-Peláez, T. J. Kwiatkowski, and K. J. Marfurt, "Generative topographic mapping for seismic facies estimation of a carbonate wash, Veracruz Basin, southern Mexico," Interpretation, vol. 2, no. 1, pp. SA31-SA47, Feb. 2014.

[11] Z. Liu, J. Cao, Y. Lu, S. Chen, and J. Liu, "A seismic facies classification method based on the convolutional neural network and the probabilistic framework for seismic attributes and spatial classification," Interpretation, vol. 7, no. 3, pp. SE225-SE236, Aug. 2019.

[12] H. Di, Z. Li, H. Maniar, and A. Abubakar, "Seismic stratigraphy interpretation by deep convolutional neural networks: A semisupervised workflow," Geophysics, vol. 85, no. 4, pp. WA77-WA86, Jul. 2020.

[13] J. S. Dramsch and M. Lüthje, "Deep-learning seismic facies on stateof-the-art CNN architectures," in Proc. 88th Annu. Int. Meeting, SEG, Expanded Abstr., Oct. 2018, pp. 1-5.

[14] D. Grana, L. Azevedo, and M. Liu, "A comparison of deep machine learning and Monte Carlo methods for facies classification from seismic data," Geophysics, vol. 85, no. 4, pp. WA41-WA52, Jul. 2020.

[15] Y. Zhang, Y. Liu, H. Zhang, and H. Xue, "Seismic facies analysis based on deep learning," IEEE Geosci. Remote Sens. Lett., vol. 17, no. 7, pp. 1119-1123, Jul. 2020.

[16] M. Liu, M. Jervis, W. Li, and P. Nivlet, "Seismic facies classification using supervised convolutional neural networks and semisupervised generative adversarial networks," Geophysics, vol. 85, no. 4, pp. O47-O58, Jul. 2020.

[17] F. Qian, M. Yin, X.-Y. Liu, Y.-J. Wang, C. Lu, and G.-M. Hu, "Unsupervised seismic facies analysis via deep convolutional autoencoders," Geophysics, vol. 83, no. 3, pp. A39-A43, May 2018.

[18] V. Das, A. Pollack, U. Wollner, and T. Mukerji, "Convolutional neural network for seismic impedance inversion," Geophysics, vol. 84, no. 6 , pp. R869-R880, Nov. 2019.

[19] M. Liu and D. Grana, "Accelerating geostatistical seismic inversion using TensorFlow: A heterogeneous distributed deep learning framework," Comput. Geosci., vol. 124, pp. 37-45, Mar. 2019.

[20] Z. Ghahramani, "Probabilistic machine learning and artificial intelligence," Nature, vol. 521, no. 7553, pp. 452-459, May 2015.

[21] S. M. Mousavi and G. C. Beroza, "Bayesian-Deep-Learning estimation of earthquake location from single-station observations," IEEE Trans. Geosci. Remote Sens., vol. 58, no. 11, pp. 8211-8224, Nov. 2020.

[22] Y. Gal and Z. Ghahramani, "Dropout as a Bayesian approximation: Representing model uncertainty in deep learning," in Proc. 33rd Int. Conf. Mach. Learn., vol. 48, Jun. 2016, pp. 1050-1059.

[23] C. Blundell, J. Cornebise, K. Kavukcuoglu, and D. Wierstra, "Weight uncertainty in neural networks," in Proc. 32nd Int. Conf. Mach. Learn., vol. 37, Jul. 2015, pp. 1613-1622.

[24] K. Shridhar, F. Laumann, and M. Liwicki, "A comprehensive guide to Bayesian convolutional neural network with variational inference," 2019, arXiv:1901.02731. [Online]. Available: http://arxiv.org/abs/1901.02731

[25] I. Goodfellow, Y. Bengio, and A. Courville, Deep Learning. Cambridge, MA, USA: MIT Press, Nov. 2016.

[26] P. Mukhopadhyay and S. Mallick, "Bayesian deep learning for seismic facies classification and its uncertainty estimation," in Proc. SEG Tech. Program Expanded Abstr., Aug. 2019, pp. 1-5.

[27] D. P. Kingma and M. Welling, "Auto-encoding variational Bayes," 2013, arXiv:1312.6114. [Online]. Available: http://arxiv.org/abs/1312.6114

[28] D. M. Blei, A. Kucukelbir, and J. D. McAuliffe, "Variational inference: A review for statisticians," J. Amer. Stat. Assoc., vol. 112, no. 518, pp. 859-877, Apr. 2017.

[29] R. Krishnan, M. Subedar, and O. Tickoo, "Specifying weight priors in Bayesian deep neural networks with empirical bayes," 2019, arXiv:1906.05323. [Online]. Available: http://arxiv.org/abs/1906.05323

[30] A. Graves, "Practical variational inference for neural networks," in Proc. 24th Int. Conf. Neural Inf. Process., vol. 24, Dec. 2011, pp. 2348-2356.

[31] Y. Wen, P. Vicol, J. Ba, D. Tran, and R. Grosse, "Flipout: Efficient pseudo-independent weight perturbations on mini-batches," Mar. 2018 arXiv:1803.04386. [Online]. Available: https://arxiv.org/abs/1803.04386

[32] A. Waldeland and A. Solberg, "3D attributes and classification of salt bodies on unlabelled datasets," in Proc. 78th Аппи. Int. Conf. Exhib., EAGE, Expanded Abstr., Jun. 2016, pp. 1-5.
[33] E. Bisong, "Google colaboratory," in Building Machine Learning and Deep Learning Models on Google Cloud Platform. Berley, CA, USA: Apress, 2019, pp. 59-64, doi: 10.1007/978-1-4842-4470-8_7.

[34] T. Zhao, F. Li, and K. J. Marfurt, "Seismic attribute selection for unsupervised seismic facies analysis using user-guided data-adaptive weights," Geophysics, vol. 83, no. 2, pp. O31-O44, Mar. 2018.

[35] R. Feng, N. Balling, and D. Grana, "Lithofacies classification of a geothermal reservoir in denmark and its facies-dependent porosity estimation from seismic inversion," Geothermics, vol. 87, Sep. 2020, Art. no. 101854, doi: 10.1016/j.geothermics.2020.101854.

[36] R. Feng, T. Mejer Hansen, D. Grana, and N. Balling, "An unsupervised deep-learning method for porosity estimation based on poststack seismic data," Geophysics, vol. 85, no. 6, pp. M97-M105, Nov. 2020.

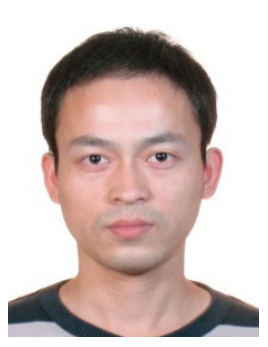

Runhai Feng received the master's degree in geophysics from Nanjing University, Nanjing, China, in 2013, and the Ph.D. degree from the Delft University of Technology, Delft, The Netherlands, in 2017.

$\mathrm{He}$ holds a post-doctoral position with Aarhus University, Aarhus, Denmark. His research interests include reservoir characterization, deep learning, and uncertainty analysis.

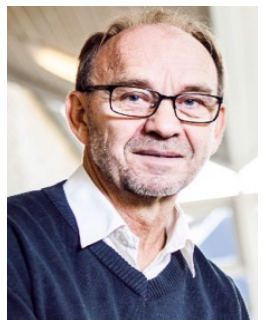

Niels Balling received the Ph.D. degree in geophysics and the D.Sc. degree from Aarhus University, Aarhus, Denmark, in 1982 and 2013, respectively.

He is also an Honorary Professor with the Department of Geoscience, Aarhus University. He has core competences within several geoscience disciplines, including controlled and natural source seismology and thermal modeling, all in relation to basic crustal and lithospheric studies, as well as geothermal energy resources.

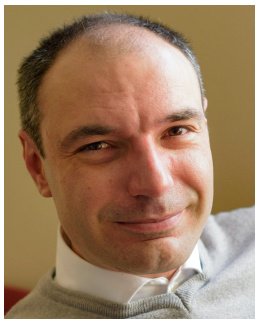

Dario Grana received the M.Sc. degree in applied mathematics from the University of Milano-Bicocca, Milan, Italy, in 2006, and the Ph.D. degree in geophysics from Stanford University, Stanford, CA, USA, in 2013.

$\mathrm{He}$ is also an Associate Professor with the Department of Geology and Geophysics, University of Wyoming, Laramie, WY, USA. His main research interests are rock physics, reservoir characterization, geostatistics, data assimilation, and inverse problems for subsurface modeling.

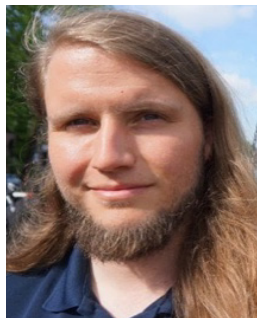

Jesper Sören Dramsch received the B.Sc. degree in geophysics and oceanography and the M.Sc. degree in geophysics from the University of Hamburg, Hamburg, Germany, in 2010 and 2014, respectively. $\mathrm{He}$ is pursuing the Ph.D. degree in 4-D seismic and machine learning with the Technical University of Denmark (DTU), Kongens Lyngby, Denmark, in collaboration with Heriot-Watt University, Edinburgh, U.K.

His main research interests lie in digital signal and image processing, geophysics, machine learning, and deep learning.

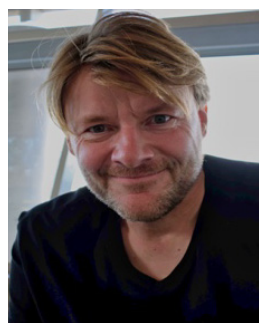

Thomas Mejer Hansen received the Ph.D. degree in geophysics from Aarhus University, Aarhus, Denmark, in 2010.

$\mathrm{He}$ is also an Associate Professor with the Department of Geoscience, Aarhus University. His main research interests are inverse problems, geostatistics, computational geoscience, and machine learning. 